ORIGINAL RESEARCH

\title{
Implementation of a Clinical Decision Support Tool to Improve Antibiotic IV-to-Oral Conversion Rates at a Community Academic Hospital
}

\author{
Tiffany Kan, Derrick Kwan, Thomas Chan, Pavani Das, and Sumit Raybardhan
}

\begin{abstract}
Background: Antibiotic IV-to-oral (IV-PO) conversion is a key initiative of antimicrobial stewardship programs. Guidelines and education are commonly described interventions to promote IV-PO conversion; however, technological interventions may be more effective in changing practice.

Objective: To determine the impact of a clinical decision support (CDS) tool on the adoption and sustainability of an antibiotic IV-PO conversion program at a community academic hospital.

Methods: A quasi-experimental study consisting of 3 phases was conducted. During phase 1 , the pre-intervention antibiotic IV-PO conversion rate was determined. During phase 2, the IV-PO conversion policy was updated, education was provided to pharmacists and physicians, and a post-intervention evaluation was conducted. During phase 3, a CDS tool was developed to generate real-time electronic alerts prompting pharmacists to assess antibiotic therapy, and post-intervention audits were performed 1 month, 3 months, and 15 months after implementation of the tool. Pantoprazole IV-PO conversion was assessed during each phase as a non-equivalent dependent variable. The primary outcome was the proportion of patients eligible for IV-PO conversion who were switched to oral therapy.

Results: Of 332 patients receiving targeted IV antibiotic therapy during the overall study period, $122(37 \%)$ met the criteria for IV-PO conversion. The phase 2 IV-PO conversion rate of 35\% (9/26) was comparable to the pre-intervention rate of $29 \%(10 / 35)(p=0.61)$. Implementation of the CDS tool significantly increased the conversion rate to $78 \%(14 / 18)$, an increase that was sustained at 3 months (71\% [17/24]) and 15 months (74\% [14/19]) after implementation $(p<0.05$ for all comparisons with phases 1 and 2). Pantoprazole conversion rates were similar across all phases.
\end{abstract}

Conclusions: Implementation of the CDS tool was effective in improving and sustaining antibiotic IV-PO conversion rates and enhancing policy compliance beyond the effects of policy revision and education. Refinement of both the policy and the tool is warranted to maximize adoption of the IV-PO conversion program.

\section{RÉSUMÉ}

Contexte: Le passage de l'antibiothérapie par voie intraveineuse (IV) à la voie orale (PO) (IV-PO) est une initiative clé des programmes de gestion des antimicrobiens. On a communément recours à des formations et à des lignes directrices pour encourager le passage d'une voie à l'autre; cependant, les interventions technologiques sont parfois plus efficaces pour favoriser le changement de pratique.

Objectif : Déterminer l'impact d'un outil d'aide à la décision clinique (ADC) sur l'adoption et la viabilité d'un programme de conversion IV-PO dans un hôpital universitaire.

Méthodes : Une étude quasi expérimentale en trois phases a été menée. La première phase a permis la détermination du taux de conversion IV-PO avant l'intervention. La deuxième phase concernait l'actualisation de la politique de conversion IV-PO, la formation des pharmaciens et médecins et la conduite d'une évaluation après l'intervention. La troisième phase a vu le développement d'un outil ADC qui génère des alertes électroniques en temps réel pour inciter les pharmaciens à évaluer l'antibiothérapie. Des évaluations ont en outre été effectuées 1 mois, 3 mois et 15 mois après la mise en place de l'outil. Le passage de l'administration du pantoprazole par voie intraveineuse (IV) à voie orale (PO) a été évalué au cours de chaque phase comme une variable dépendante non équivalente. Le résultat principal fut la proportion de patients admissibles à la conversion IV-PO qui ont été orientés vers un traitement par voie orale.

Résultats : Des 332 patients recevant une antibiothérapie ciblée par voie intraveineuse (IV) pendant l'étude, 122 (37\%) répondaient au critère de la conversion IV-PO. Le taux de conversion IV-PO de $35 \%$ (9/26) de la phase 2 était comparable au taux avant l'intervention de $29 \%$ (10/35) $(p=0,61)$. La mise en place de l'outil ADC a grandement augmenté le taux de conversion, qui est passé à $78 \%$ (14/18) : une augmentation maintenue trois mois $(71 \%$ [17/24]) et 15 mois (74 \% [14/19]) après la mise en place $(p<0,05$ par rapport aux phases 1 et 2$)$. Les taux de conversion du pantoprazole étaient similaires durant toutes les phases.

Conclusions : La mise en place de l'outil ADC a permis d'améliorer et de maintenir les taux de conversion IV-PO et de renforcer le respect des 
Keywords: antimicrobial stewardship, clinical decision support systems, IV-to-oral conversion, antimicrobials, pharmacists

Can J Hosp Pharm. 2019;72(6):455-61 politiques au-delà des effets de la révision des politiques et de la formation à celles-ci. Le perfectionnement de la politique et de l'outil se justifie pour maximiser l'adoption du programme de conversion IV-PO.

Mots-clés : gestion de l'utilisation des antimicrobiens, systèmes d'aide à la décision clinique, passage de la voie intraveineuse (IV) à la voie orale (PO), antimicrobiens, pharmaciens

\section{INTRODUCTION}

A ntibiotic IV-to-oral (IV-PO) conversion is a key initiative of antimicrobial stewardship programs. Transitioning patients from IV to oral antibiotics when it is appropriate to do so has several advantages, including decreasing the risk of catheterrelated infections, shortening the length of hospital stay, and decreasing health care costs. ${ }^{1-4}$ Considering the clinical and cost benefits, IV-PO conversion programs are strongly recommended by antimicrobial stewardship implementation guidelines ${ }^{5,6}$ and are promoted in the Choosing Wisely Canada campaign. ${ }^{7}$

Guidelines, education, and non-technological interventions have traditionally been the primary means of facilitating IV-PO conversion $^{8-11}$; however, technological interventions based on human factors engineering principles are viewed as more effective in changing behaviours and practices. ${ }^{12}$ There is a growing call for the integration of computerized clinical decision support (CDS) systems in antimicrobial stewardship programs to improve antibiotic prescribing practices and to enhance the implementation and sustainability of initiatives. ${ }^{5,7,13,14}$ These systems use patient data and clinical knowledge to provide patient-specific recommendations that aid health care providers in clinical decision-making at the point of care. ${ }^{13}$ Therefore, guidelines and education paired with CDS tools could represent a more effective approach to promoting IV-PO conversion.

A quality improvement study, with the ultimate goal of enhancing adoption of an IV-PO conversion program, was undertaken between 2013 and 2016 at a community academic hospital. At the study institution, a pharmacist-initiated, criteriabased IV-PO conversion policy had been in place since 2007 . The current study involved a stepwise approach, beginning with an update to the policy accompanied by staff education, followed by development and implementation of a CDS tool, with evaluation of IV-PO conversion rates after each intervention. The objective of this study was to determine the impact of the CDS tool on the adoption and sustainability of the antibiotic IV-PO conversion program at the study institution.

\section{METHODS}

\section{Study Design}

A quasi-experimental study was conducted at a 420-bed community academic hospital in Toronto, Ontario, Canada. The institution is a single-site hospital that provides a range of acute care and ambulatory services, with an off-site long-term care centre. The hospital uses an electronic medical record system (Cerner Corporation, Kansas City, Missouri) that integrates computerized physician order entry, medication administration, clinical documentation, and CDS capabilities. The hospital has an established antimicrobial stewardship program involving an infectious diseases physician (P.D.) and 2 pharmacists (T.K., S.R.), who perform prospective audit and feedback for hospital inpatients within the medicine program.

The study consisted of 3 phases: the pre-intervention period (July and August 2013); policy revision and staff education, with post-intervention evaluation (May and June 2014); and development and implementation of a CDS tool, with postintervention evaluation (October 2014 to January 2016). Adult patients ( $\geq 18$ years) admitted on 5 general medicine and surgical units were reviewed for eligibility and inclusion in the study. The study protocol received approval from the institutional research ethics board.

\section{Phase 1: Pre-intervention}

The first iteration of the IV-PO conversion policy at the hospital was introduced in 2007. The policy covers 9 targeted IV antibiotic agents (Table 1) and 2 non-antibiotic agents (pantoprazole and ranitidine). The policy allows for pharmacists to automatically switch a targeted IV antibiotic agent to oral therapy if specific criteria are met (Table 2). The criteria for IV-PO conversion of the non-antibiotic agents are the patient being able to tolerate oral medications and the patient continuing to need the medication for a specified indication. A baseline (pre-intervention) assessment of the antibiotic IV-PO conversion rate was conducted. 
Table 1. Targeted IV Antibiotics with Corresponding Oral Antibiotic after Conversion

\begin{tabular}{ll} 
IV Antibiotic & $\begin{array}{c}\text { Oral Antibiotic } \\
\text { after Conversion }\end{array}$ \\
\hline ampicillin & amoxicillin \\
\hline azithromycin & azithromycin \\
\hline cefazolin & cephalexin \\
\hline cefuroxime & cefuroxime \\
\hline ciprofloxacin & ciprofloxacin \\
\hline clindamycin & clindamycin \\
\hline levofloxacin & levofloxacin \\
\hline metronidazole & metronidazole \\
\hline penicillin G & penicillin VK \\
\hline
\end{tabular}

\section{Phase 2: Policy Revision and Staff Education}

The next phase of the study focused on revision of the IVPO policy to reflect workflow changes and provision of staff education to improve adoption of the IV-PO program. Implementation of the policy in 2007 occurred before establishment of an electronic medical record system at the hospital, which occurred in 2010; therefore, the policy required revisions to reflect the transition from a paper-based practice to an electronic workflow. Specifically, procedures for electronic documentation of pharmacists' assessments of IV-PO conversion for individual patients and electronic communication of these assessments to the health care team were incorporated into the policy, with approval from the institution's pharmacy and therapeutics committee. Apart from these revisions in the procedure, the policy remained the same; specifically, there were no changes to eligibility criteria or the targeted IV medication list. Staff education was then provided to both pharmacists and physicians to increase policy awareness and improve policy adoption. The antimicrobial stewardship pharmacists delivered an education session to the pharmacists and provided a pocket reference guide reviewing the principles of IV-PO conversion and the institution's IV-PO policy. The antimicrobial stewardship pharmacists also delivered a separate presentation to physicians within the medicine program regarding the general principles of IV-PO conversion and the institutional policy. A post-intervention audit of the IV-PO conversion rate was performed 1 month after the delivery of education. Pharmacists' and physicians' knowledge and awareness of IV-PO conversion were not directly assessed.

\section{Phase 3: Development and Implementation of CDS Tool}

Following the delivery of staff education and assessment of its effectiveness, a CDS tool to facilitate antibiotic IV-PO conversion was developed by the antimicrobial stewardship pharmacists, working in collaboration with a consultant from the hospital's clinical informatics team. This criteria-based tool, which is embedded within the Cerner electronic medical record system, identifies patients who are potentially eligible for antibiotic IV$\mathrm{PO}$ conversion and generates real-time electronic alerts to prompt a pharmacist's assessment. An alert is generated if all of the following criteria are met: the patient is receiving a targeted IV antibiotic, the antibiotic order has been active for at least $48 \mathrm{~h}$, the antibiotic order does not have a fixed duration, there is no documented fever (i.e., no temperature measurements $>37.6^{\circ} \mathrm{C}$ ) in the past $24 \mathrm{~h}$, and the patient has an order for an oral diet. The third criterion, which excludes orders with a fixed duration, was a practical consideration to reduce the risk of alert fatigue. At the study hospital, most antibiotic orders with a fixed duration are for surgical prophylaxis; these orders generally have a fixed duration of $24 \mathrm{~h}$ and are automatically discontinued after this period has elapsed. The exclusion of this type of order was intended to improve the specificity of the CDS alert.

Once all of the criteria are met for a particular patient, a realtime electronic alert is automatically generated and integrated into the unit pharmacist's daily electronic task list. The unit pharmacist then assesses the patient and proceeds with IV-PO conversion if appropriate. Before hospital-wide implementation of the CDS tool, an education session was provided to the pharmacists to discuss features of the CDS tool and the associated workflow. Post-intervention audits were performed at 1 month (phase $3 \mathrm{a}$ ), 3 months (phase 3b), and 15 months (phase 3c) after CDS implementation to determine the tool's impact on the sustainability of the IV-PO conversion program.

\section{Table 2. Criteria for Pharmacist-Initiated Antibiotic IV-to-Oral Conversion*}

Inclusion Criteria

All inclusion criteria must be met for the patient to be eligible for IV-to-oral conversion:

- The patient has a functioning gastrointestinal tract and is tolerating oral medications

- The patient is showing clinical improvement and has been afebrile for $24 \mathrm{~h}$

- The patient continues to need the antibiotic for treatment of the infectious disease (as documented in the patient's medical chart by the most responsible physician)

\section{Exclusion Criteria}

The presence of any exclusion criterion would result in patient's exclusion from eligibility for IV-to-oral conversion:

- The patient has an infectious disease for which oral therapy would be inappropriate (e.g., endocarditis, Staphylococcus aureus bacteremia, meningitis)

- The patient has a "nothing by mouth" order

- The patient has neutropenia

₹The criteria outlined here were in place before the current study began. 


\section{Data Collection}

During each phase of the study, chart audits were conducted over a 6-week evaluation period on 5 general medicine and surgical units. All patients on these units who were receiving targeted IV antibiotics were included in the audits. The following de-identified data were collected: patient age and sex, type of infectious disease, and current antibiotic regimen. Eligibility for IV-PO conversion was determined according to the policy's criteria, and conversion to oral therapy was checked. Pantoprazole IV-PO conversion was assessed concurrently during each phase as a non-equivalent dependent variable. Patients receiving IV pantoprazole were identified, their eligibility for IV-PO conversion was determined, and conversion to oral therapy was checked.

\section{Outcomes and Statistical Analysis}

Descriptive statistics were calculated for all variables of interest. Categorical variables were summarized using counts and percentages. Continuous variables were summarized as the mean with standard deviation or as the median with interquartile range (IQR).

The primary outcome was the proportion of patients eligible for antibiotic IV-PO conversion who were switched to oral therapy. The secondary outcome was the duration of IV therapy until the switch to oral therapy. A non-equivalent dependent variable, pantoprazole IV-PO conversion, was included in the study to increase the validity of the results. ${ }^{15}$ Pantoprazole was a targeted medication under the institutional IV-PO conversion policy. However, the study interventions (staff education and CDS implementation) were not directed at improving conversion to oral therapy for non-antibiotic medications. Instead, staff education focused on antibiotic IV-PO conversion, and the CDS tool assessed the potential for IV-PO conversion for targeted antibiotics only. Therefore, it was hypothesized that rates of antibiotic IV-PO conversion would increase following the interventions, whereas rates of pantoprazole IV-PO conversion would remain unchanged.

Categorical variables were compared using the $\chi^{2}$ test or the Fisher exact test. Continuous variables were compared using the $t$ test. All effects were considered significant at $p$ less than 0.05 . All statistical analyses were conducted using SAS version 9.4 software (SAS Institute, Cary, North Carolina).

\section{RESULTS}

Over the entire study, a total of 332 patients who were receiving targeted IV antibiotics were reviewed for eligibility for IV-PO conversion, of whom 122 patients (37\%) met the eligibility criteria; baseline characteristics of these patients are summarized in Table 3.

Antibiotic IV-PO conversion rates during all phases are shown in Figure 1. During phase 1, the pre-intervention phase, 10 (29\%) of 35 eligible patients were switched to oral therapy. Following policy revision and staff education (phase 2), 9 (35\%) of 26 eligible patients were switched to oral therapy, which was comparable to the rate in phase $1(p=0.61)$. Following implementation of the CDS tool, the proportion of eligible patients switched to oral therapy increased significantly to 14 (78\%) of 18 patients at 1 month after implementation (phase 3a: $p=0.001$ compared with phase $1 ; p=0.006$ compared with phase 2 ). This

\section{Table 3. Baseline Characteristics of Patients Who Met Eligibility Criteria for Antibiotic IV-to-Oral Conversion}

Phase; No. (\%) of Patients*

\begin{tabular}{|c|c|c|c|c|c|c|c|c|c|c|}
\hline \multirow{3}{*}{$\begin{array}{l}\text { Variable } \\
\text { Age (years) (median and IQR) } \\
\text { Sex female }\end{array}$} & \multicolumn{2}{|c|}{$\begin{array}{l}\text { Phase 1: } \\
\text { Pre-intervention } \\
(n=35)\end{array}$} & \multicolumn{2}{|c|}{$\begin{array}{l}\text { Phase 2: } \\
\text { Policy and } \\
\text { Education } \\
(n=26)\end{array}$} & \multicolumn{2}{|c|}{$\begin{array}{c}\text { Phase 3a: } \\
1 \text { month } \\
\text { after CDS } \\
(n=18)\end{array}$} & \multicolumn{2}{|c|}{$\begin{array}{c}\text { Phase 3b: } \\
3 \text { months } \\
\text { after CDS } \\
(n=24)\end{array}$} & \multicolumn{2}{|c|}{$\begin{array}{c}\text { Phase 3c: } \\
15 \text { months } \\
\text { after CDS } \\
(n=19)\end{array}$} \\
\hline & 72 & $(55-84)$ & 73 & $(59-87)$ & 86 & $(78-89)$ & 71 & $(59-88)$ & 79 & $(52-88)$ \\
\hline & 15 & $(43)$ & 13 & $(50)$ & 8 & $(44)$ & 21 & $(88)$ & 11 & $(58)$ \\
\hline \multicolumn{11}{|l|}{ Infectious disease } \\
\hline Respiratory & 15 & (43) & 6 & (23) & 3 & (17) & 13 & (54) & 4 & $(21)$ \\
\hline Intra-abdominal & 13 & (37) & 7 & $(27)$ & 5 & (28) & 5 & (21) & 7 & (37) \\
\hline Skin and soft tissue & 6 & (17) & 5 & (19) & 7 & (39) & 3 & (13) & 3 & (16) \\
\hline Urinary & & (3) & $\varepsilon$ & (31) & 3 & $(17)$ & 3 & (13) & 5 & (26) \\
\hline Targeted IV antibiotict & \multicolumn{2}{|c|}{$n=38$} & \multicolumn{2}{|c|}{$n=30$} & \multicolumn{2}{|c|}{$n=20$} & \multicolumn{2}{|c|}{$n=25$} & \multicolumn{2}{|c|}{$n=22$} \\
\hline Ampicillin & 0 & & 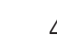 & (13) & 1 & (5) & 1 & (4) & 3 & $(14)$ \\
\hline Azithromycin & 6 & (16) & 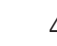 & (13) & 1 & (5) & 9 & (36) & 1 & (5) \\
\hline Cefazolin & 8 & (21) & 11 & (37) & 8 & $(40)$ & 4 & (16) & 6 & (27) \\
\hline Ciprofloxacin & 2 & (5) & 3 & (10) & 3 & (15) & 2 & (8) & 3 & (14) \\
\hline Levofloxacin & 8 & (21) & 2 & (7) & 2 & (10) & 4 & (16) & 2 & (9) \\
\hline Metronidazole & 14 & (37) & 6 & (20) & 5 & $(25)$ & 5 & (20) & 7 & (32) \\
\hline
\end{tabular}

CDS = clinical decision support, IQR = interquartile range

*Except where indicated otherwise.

tPatients may have been receiving more than 1 targeted IV antibiotic concurrently; percentages in this section are calculated in relation to the total number of antibiotic prescriptions. 


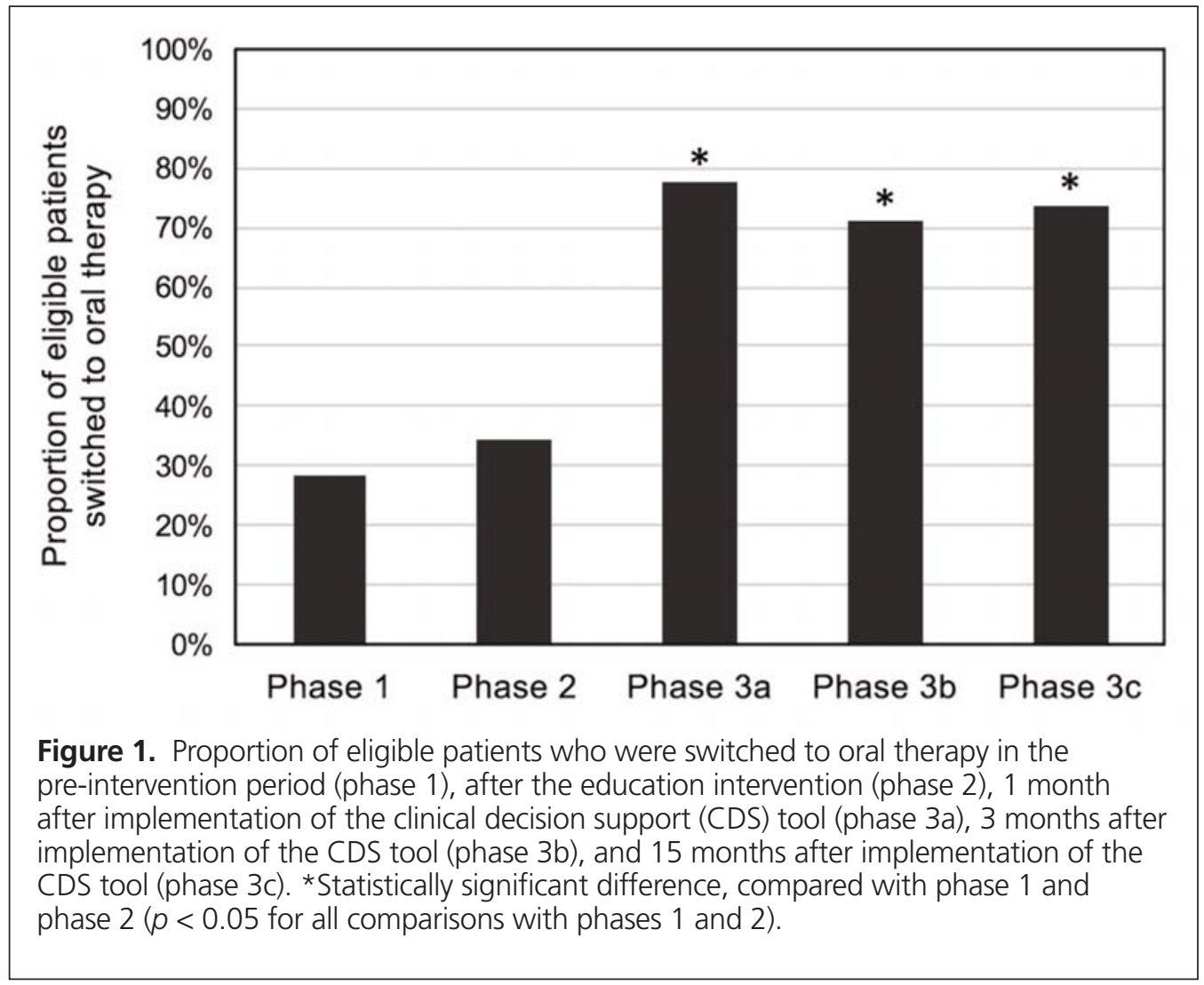

improvement was sustained with 17 (71\%) of 24 patients being switched to oral therapy at 3 months after CDS implementation, and $14(74 \%)$ of 19 patients being switched to oral therapy at 15 months after implementation (phases $3 b$ and $3 c$, respectively: $p=0.001$ for both compared with phase $1 ; p=0.01$ for both compared with phase 2). All of the patients who were switched to oral therapy completed their antibiotic course with oral therapy and did not require transition back to IV therapy. With regard to the non-equivalent dependent variable, pantoprazole IV-PO conversion rates were similar across all phases: 18 (58\%) of 31 patients in phase $1,19(63 \%)$ of 30 patients in phase 2 , $33(69 \%)$ of 48 patients in phase $3 \mathrm{a}, 12(60 \%)$ of 20 patients in phase $3 \mathrm{~b}$, and $17(63 \%)$ of 27 patients in phase $3 \mathrm{c}$ (all $p>0.30$ compared with phase 1). The median duration of IV antibiotic therapy before IV-PO conversion was similar across all phases: 3 days (IQR 3-4) in phases 1, 2, 3a, and 3b, and 4 days (IQR 35) in phase $3 c$.

During the CDS implementation phase (phase 3), electronic alerts were generated for 186 patients, of whom 61 (33\%) fulfilled all criteria for IV-PO conversion. The main reasons for ineligibility were the type of infectious disease precluding pharmacist-initiated IV-PO conversion (for 55 patients) and the antibiotic no longer being indicated (for 36 patients). The median duration from initiation of IV antibiotic therapy to generation of an electronic alert was 2 days. For eligible patients in this phase, a total of 67 courses of targeted IV antibiotic therapy met conversion criteria, of which 17 were not converted (8 courses of metronidazole, 5 courses of azithromycin, and 4 courses of cefazolin).

\section{DISCUSSION}

In this study, the implementation of a CDS tool to facilitate antibiotic IV-PO conversion was associated with a doubling of IV-PO conversion rates relative to the combination of a policy update and education. Pantoprazole IV-PO conversion rates were similar across all phases, which suggests that the improvement in antibiotic conversion was attributable to implementation of the CDS tool. Antimicrobial stewardship activities, such as prospective audit and feedback, remained unchanged during the study period, further supporting the conclusion that improvements in compliance were attributable to the CDS tool.

There is a lack of comparative data to indicate the type of intervention that is most effective in optimizing and sustaining IV-PO conversion programs. This is the first study of which we are aware that assessed the effectiveness of stepwise implementation of a pair of non-technological interventions (policy implementation and staff education) and a technological intervention. We observed only minimal improvements in IV-PO conversion rates with the policy update and associated education, whereas the technological intervention proved far more 
effective in improving program uptake. In addition, this study demonstrated long-term program sustainability with use of the CDS tool, with sustained improvements in IV-PO conversion rates at 3 and 15 months after implementation. Previous studies have documented successes with guideline implementation, education, and printed checklists and information sheets ${ }^{8-11}$; however, data supporting the long-term sustainability of these interventions are lacking. One study that used guidelines and education was unable to demonstrate a sustained improvement in IV-PO conversion rates 3 months after implementation. ${ }^{16}$ Person-based strategies, such as policies and education, that are implemented in isolation often do not produce long-term sustainable benefits, and system-based strategies may be required. ${ }^{17}$ Therefore, implementation of CDS tools complementary to policy and education may be necessary to achieve sustained improvements.

Despite the multipronged approach to improving IV-PO program uptake, including CDS implementation, IV-PO conversion rates remained steady during phase 3 of our study, at about $75 \%$. This may point to a need to further refine the IV-PO policy. For example, the policy currently outlines infectious disease syndromes for which IV-PO conversion is inappropriate; however, there may be a lack of clarity regarding the clinical situations that are suitable for conversion. Specifying the indications for which IV-PO conversion would be appropriate may lead to better policy compliance. Another reason for not transitioning eligible patients to oral therapy was use of combination antibiotic regimens in which only one of the antibiotics was covered by the IV-PO policy. Azithromycin and metronidazole were both included in the list of antibiotics targeted for conversion in the institutional policy, but in most cases of non-conversion of these 2 antibiotics in phase 3 (11/13), conversion did not occur because the azithromycin or metronidazole was being administered in combination with ceftriaxone, which is not included in the list of targeted antibiotics. Although combination antibiotic therapy was not a criterion for exclusion from IV-PO conversion, pharmacists may have been hesitant to partially convert these regimens (by changing only 1 of the 2 drugs from IV to oral administration). Recent antimicrobial stewardship recommendations promote interventions for certain infectious disease syndromes, ${ }^{5,6}$ and we are considering adding syndromespecific IV-PO conversion recommendations into the policy to further improve conversion rates.

There is growing interest in leveraging technology to facilitate and enhance sustainability of antimicrobial stewardship initiatives. Previous studies have described computerized IV-PO alerts, which generally assess whether a patient is receiving a targeted medication and whether medications or diet is being administered orally. ${ }^{18-20}$ More recently, a physician-targeted CDS alert that assessed more parameters, including presence of fever and neutrophilia, has been described. This intervention was associated with a decrease in duration of IV therapy. ${ }^{21}$ Our CDS tool similarly incorporated more complex rules to identify with greater specificity those patients who are eligible for IV-PO conversion; it also targeted pharmacists rather than physicians. Pharmacists play a central role in medication review and IV-PO conversion, and previous studies have demonstrated the effectiveness of pharmacist-managed IV-PO conversion programs. ${ }^{3,4}$

We are continuing to refine the CDS tool to enhance its effectiveness and usability. Our analysis showed that only $33 \%$ of patients for whom an electronic alert was generated fulfilled all criteria for IV-PO conversion, which raises a concern about alert fatigue. ${ }^{14}$ The main reasons for ineligibility for IV-PO conversion were presence of a type of infectious disease that precluded pharmacist-initiated IV-PO conversion (as specified by the policy's exclusion criteria) and antibiotics no longer being indicated. There is increasing interest in indication-based prescribing to improve medication safety and prescribing practices. ${ }^{22}$ Our institution currently does not incorporate the indication into medication orders; however, inclusion of such a parameter could improve the CDS tool's specificity, decrease the proportion of non-actionable alerts, and reduce the risk of alert fatigue. Other considerations for improvement involve reassessing the criteria for generating an alert. The CDS tool currently excludes fixed-duration antibiotic orders; however, this exclusion could lead to potentially eligible IV antibiotic orders being overlooked, especially with antimicrobial stewardship programs' increasing emphasis on optimizing antibiotic durations. ${ }^{5}$ Removing this exclusion could potentially increase the sensitivity of the tool. Furthermore, it was noted that the time to IV-PO conversion remained unchanged despite the study interventions, whereas previous studies have noted significant reductions in the duration of IV therapy before conversion..$^{2-4,8-11}$ In our CDS tool, the alert was generated when the antibiotic order had been active for at least $48 \mathrm{~h}$, a threshold that was based on discussions with clinicians and a recognized timeframe for antimicrobial reassessment. ${ }^{6}$ Shortening this timeframe for alert generation is a potential strategy to reduce the time to conversion.

Our study had several limitations. First, the study had a relatively small sample size, which was attributable to the short (6-week) evaluation periods (which were due, in turn, to resource limitations). Also, we did not account for potential seasonal variation in antibiotic prescribing because of the staggered time periods for each phase. Furthermore, our study was subject to the limitations inherent to quasi-experimental study designs, such as lack of randomization and difficulty controlling the confounding variables; however, we incorporated a non-equivalent dependent variable to increase the study validity. ${ }^{15,23}$ In terms of the study outcomes, we focused on those that informed implementation and sustainability of the IV-PO conversion program, rather than on clinical and economic outcomes; benefits in these areas have been demonstrated in previous studies. ${ }^{14,8-11}$ Indeed, it would have 
This single copy is for your personal, non-commercial use only.

For permission to reprint multiple copies or to order presentation-ready copies for distribution, contact CJHP at publications@cshp.pharmacy

been difficult to interpret clinical outcomes in this study, because of the aforementioned small sample size and difficulty in controlling for confounding factors. Finally, by their nature, chart reviews rely on documentation in existing medical records, which raises the risk of incomplete documentation and missing data.

\section{CONCLUSION}

The results of this study showed that implementation of a criteria-based CDS tool was effective in improving and sustaining antibiotic IV-PO conversion rates, while enhancing policy compliance beyond the effect of policy revision combined with education. Implementation of such a tool could be considered at other institutions to optimize antibiotic IV-PO conversion. Further refinement of the policy and the CDS tool is warranted to maximize antibiotic IV-PO program adoption.

\section{References}

1. Goff DA, Bauer KA, Reed EE, Stevenson KB, Taylor JJ, West JE. Is the "low-hanging fruit" worth picking for antimicrobial stewardship programs? Clin Infect Dis. 2012;55(4):587-92.

2. Davis SL, Delgado G, McKinnon PS. Pharmacoeconomic considerations associated with the use of intravenous-to-oral moxifloxacin for communityacquired pneumonia. Clin Infect Dis. 2005;41 Suppl 2:S136-43.

3. Kuti JL, Le TN, Nightingale CH, Nicolau DP, Quintiliani R. Pharmacoeconomics of a pharmacist-managed program for automatically converting levofloxacin route from i.v. to oral. Am J Health Syst Pharm. 2002; 59(22):2209-15.

4. Przybylski KG, Rybak MJ, Martin PR, Weingarten CM, Zaran FK, Stevenson JG, et al. A pharmacist-initiated program of intravenous to oral antibiotic conversion. Pharmacotherapy. 1997;17(2):271-6.

5. Barlam TF, Cosgrove SE, Abbo LM, MacDougall C, Schuetz AN, Septimus EJ, et al. Implementing an antibiotic stewardship program: guidelines by the Infectious Diseases Society of America and the Society of Healthcare Epidemiology of America. Clin Infect Dis. 2016;62(10):e51-77.

6. Centers for Disease Control and Prevention. Core elements of hospital antibiotic stewardship programs. Atlanta (GA): US Department of Health and Human Services, Centers for Disease Control and Prevention; 2014 [cited 2019 Feb 18]. Available from: http://www.cdc.gov/getsmart/healthcare/ implementation/core-elements.html

7. Leis JA, Evans GA, Ciccotelli W, Garber G, Gregson D, Lee TC, et al. Choosing Wisely Canada - top five list in infectious diseases: an official position statement of the Association of Medical Microbiology and Infectious Disease (AMMI) Canada. J Assoc Med Microbiol Infect Dis Can. 2016;1(1): $5-11$

8. Mertz D, Koller M, Haller P, Lampert ML, Plagge H, Hug B, et al. Outcomes of early switching from intravenous to oral antibiotics on medical wards. J Antimicrob Chemother. 2009;64(1):188-99.

9. McLaughlin CM, Bodasing N, Boyter AC, Fenelon C, Fox JG, Seaton RA. Pharmacy-implemented guidelines on switching from intravenous to oral antibiotics: an intervention study. QJ Med. 2005;98(10):745-52.

10. Martinez MJ, Freire A, Castro I, Inaraja MT, Ortega A, Del Campo V, et al. Clinical and economic impact of a pharmacist-intervention to promote sequential intravenous to oral clindamycin conversion. Pharm World Sci. 2000;22(2):53-8.

11. Sevinc F, Prins JM, Koopmans RP, Langendijk PN, Bossuyt PM, Dankert J, et al. Early switch from intravenous to oral antibiotics: guidelines and implementation in a large teaching hospital. J Antimicrob Chemother. 1999; 43(4):601-6.

12. Institute for Safe Medication Practices. Medication error prevention "toolbox". ISMP Med Saf Alert. 1999 Jun 2 [cited 2019 Nov 7]. Available from: https://www.ismp.org/resources/medication-error-prevention-toolbox
13. Pestotnik SL. Expert clinical decision support systems to enhance antimicrobial stewardship programs: insights from the Society of Infectious Diseases Pharmacists. Pharmacotherapy. 2005;25(8):1116-25.

14. Forrest GN, Van Schooneveld TC, Kullar R, Schulz LT, Duong P, Postelnick M. Use of electronic health records and clinical decision support systems for antimicrobial stewardship. Clin Infect Dis. 2014;59 Suppl 3:S122-33.

15. Harris AD, Bradham DD, Baumgarten M, Zuckerman IH, Fink JC, Perencevich EN. The use and interpretation of quasi-experimental studies in infectious diseases. Clin Infect Dis. 2004;38(11):1586-91.

16. van Niekerk AC, Venter DJ, Boschmans SA. Implementation of intravenous to oral antibiotic switch therapy guidelines in the general medical wards of a tertiary-level hospital in South Africa. J Antimicrob Chemother. 2012;67(3): 756-62.

17. Institute for Safe Medication Practices Canada. Designing effective recommendations. Ont Crit Incident Learn. 2013 [cited 2019 Nov 7]; 4:1-2. Available from: https://www.ismp-canada.org/download/ocil/ ISMPCONCIL2013-4_EffectiveRecommendations.pdf

18. Galanter W, Liu XF, Lambert BL. Analysis of computer alerts suggesting oral medication use during computerized order entry of i.v. medications. Am J Health Syst Pharm. 2010;67(13):1101-5.

19. Fischer MA, Solomon DH, Teich JM, Avorn J. Conversion from intravenous to oral medications: assessment of a computerized intervention for hospitalized patients. Arch Intern Med. 2003;163(21):2585-9.

20. Glemaud I. Use of a physician order entry system to identify opportunities for intravenous to oral levofloxacin conversion. Am J Health Syst Pharm 2000;57 Suppl 3:S14-6.

21. Beeler PE, Kuster SP, Eschmann E, Weber R, Blaser J. Earlier switching from intravenous to oral antibiotics owing to electronic reminders. Int J Antimicrob Agents. 2015;46(4):428-33.

22. Schiff GD, Seoane-Vazquez E, Wright A. Incorporating indications into medication ordering - time to enter the age of reason. $N$ Engl J Med. 2016; 375(4):306-9.

23. Harris AD, Lautenbach E, Perencevich E. A systematic review of quasiexperimental study designs in the fields of infection control and antibiotic resistance. Clin Infect Dis. 2005;41(1):77-82.

Tiffany Kan, BSCPhm, PharmD, BCPS, RPh, is with the Department of Pharmacy, North York General Hospital, and the Leslie Dan Faculty of Pharmacy, University of Toronto, Toronto, Ontario.

Derrick Kwan, BScPhm, ACPR, RPh, is with the Department of Clinical Informatics, North York General Hospital, Toronto, Ontario.

Thomas Chan, BSCPhm, MBA, RPh, was, at the time of this study, with the Department of Pharmacy, North York General Hospital, Toronto, Ontario. He is now with the Department of Pharmacy, Centre for Addiction and Mental Health, Toronto, Ontario

Pavani Das, MD, is with the Division of Infectious Diseases, Department of Medicine, North York General Hospital, Toronto, Ontario.

Sumit Raybardhan, BSCPhm, ACPR, MPH, RPh, is with the Department of Pharmacy, North York General Hospital, Toronto, Ontario.

Competing interests: None declared.

\section{Address correspondence to:}

Dr Tiffany Kan

Department of Pharmacy

North York General Hospital

4001 Leslie Street

Toronto ON M2K 1E1

e-mail: tiffany.kan@nygh.on.ca

Funding: None received. 